\title{
A large cervical osteophyte causing dysphagia in an elderly patient
}

\author{
Giuseppe Rusignuolo ${ }^{a}$, Henning Schwacha ${ }^{a}$, Arthur Schmidt ${ }^{a}$, Dominik Bettinger ${ }^{a, b}$ \\ University of Freiburg, Germany
}

A 72-year-old man presented to our department with progressive dysphagia and difficulty in eating any solid food. The patient reported a weight loss of $20 \mathrm{~kg}$. The patient's previous medical history revealed nicotine abuse and adenocarcinoma of the right lower lung lobe, treated successfully by lobectomy 6 years before. Follow-up examinations confirmed complete tumor response. The physical examination revealed no significant findings. Upper gastrointestinal endoscopy was performed, revealing a significant extraluminal compression of the upper esophagus in the absence of any intraluminal pathological findings (Fig. 1). Furthermore, cricopharyngeal achalasia and Zenker's diverticulum were excluded.

Considering the patient's medical history, a tumor recurrence seemed likely. A computed tomography scan of the chest was thus performed, which instead showed a large osteophyte deriving from the second and third thoracic vertebra, leading to a strong compression of the esophagus (Fig. 2A). In addition, a barium swallow examination was performed, which confirmed that the large osteophyte was indeed causing an impaired esophageal passage resulting in dysphagia (Fig. 2B).

This is a rare and uncommon cause of dysphagia in an elderly patient. Besides the common causes of dysphagia, such as neoplasia, peptic strictures and achalasia, anterior osteophytes of the spine can be one of the differential diagnoses [1-2]. Large osteophytes have been associated with dysphagia, and surgery is indicated in patients with persisting and progressing symptoms. Good clinical results after surgery have been reported; however, in some cases dysphagia may persist, probably because of irreversible changes in the surrounding tissue [3].

${ }^{a}$ Department of Medicine II, Medical Center University of Freiburg, Faculty of Medicine; berta-Ottenstein Programme, Faculty of Medicine, University of Freiburg, Freiburg, Germany

Conflict of Interest: DB is supported by the Berta-Ottenstein Programme of the Faculty of Medicine, University of Freiburg. The other authors have nothing to declare with regard to this work.

Correspondence to: Dr. Dominik Bettinger, Medical Center University of Freiburg, Department of Medicine II, Hugstetter Str. 55, D-79106 Freiburg, Germany, e-mail: dominik.bettinger@uniklinik-freiburg.de

Received 28 May 2020; accepted 31 May 2020;

published online 30 June 2020

DOI: https://doi.org/10.20524/aog.2020.0509

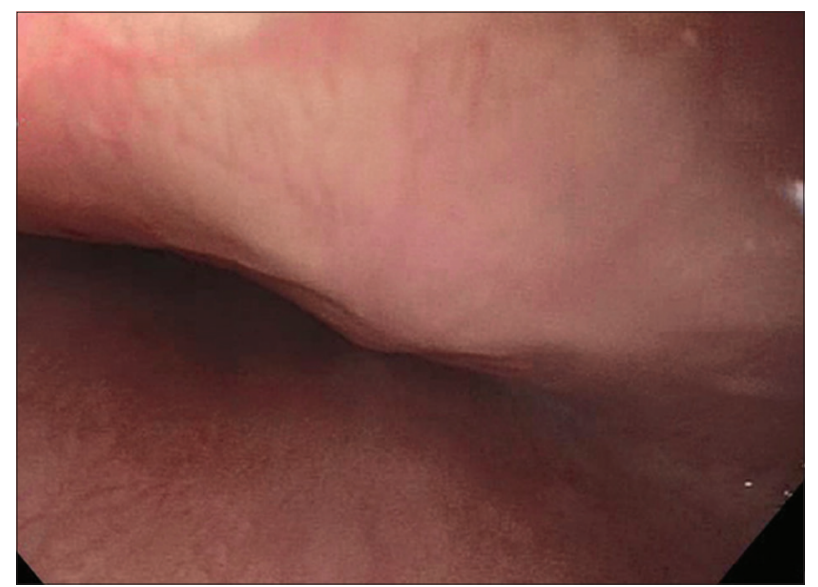

Figure 1 Upper gastrointestinal endoscopy showed extraluminal compression of the esophagus, not reversed after $\mathrm{CO}_{2}$ insufflation. With slight pressure the stenosis was passed with the endoscope
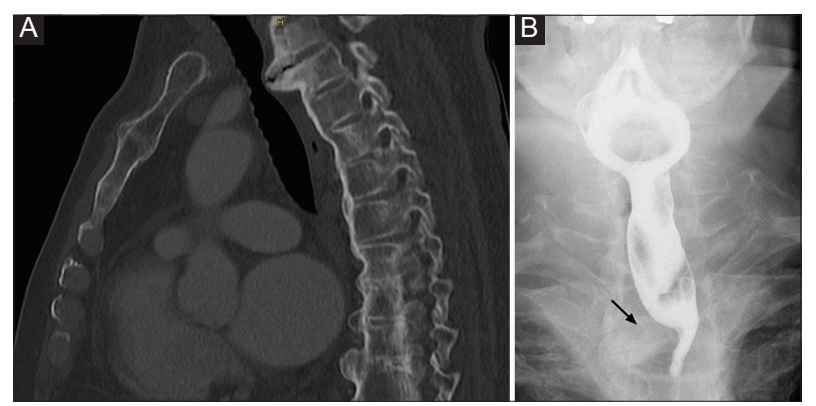

Figure 2 Chest computed tomography scan (A) and barium swallow examination (B) showed a large cervical osteophyte as the reason for the esophageal compression

\section{References}

1. Malagelada JR, Bazzoli F, Boeckxstaens G, et al. World gastroenterology organisation global guidelines: dysphagiaglobal guidelines and cascades update September 2014. J Clin Gastroenterol 2015;49:370-378.

2. Cho SY, Choung RS, Saito YA, et al. Prevalence and risk factors for dysphagia: a USA community study. Neurogastroenterol Motil 2015;27:212-219.

3. Jeong H, Seo HG, Han TR, Chung CK, Oh BM. Kinematic changes in swallowing after surgical removal of anterior cervical osteophyte causing dysphagia: a case series. Ann Rehabil Med 2014;38:865-870. 